ARTICLE

\title{
Two nuclear effectors of the rice blast fungus modulate host immunity via transcriptional reprogramming
}

Seongbeom Kim (1) 1, Chi-Yeol Kim¹, Sook-Young Park², Ki-Tae Kim (1) 1, Jongbum Jeon (1) 3, Hyunjung Chung 1,9, Gobong Choi ${ }^{3}$, Seomun Kwon ${ }^{1,10}$, Jaeyoung Choi ${ }^{1,11}$, Junhyun Jeon ${ }^{4}$, Jong-Seong Jeon (1) ${ }^{5}$, Chang Hyun Khang ${ }^{6}$, Seogchan Kang ${ }^{7}$ \& Yong-Hwan Lee (i) ${ }^{1,3,8}$ 瓜

Pathogens utilize multiple types of effectors to modulate plant immunity. Although many apoplastic and cytoplasmic effectors have been reported, nuclear effectors have not been well characterized in fungal pathogens. Here, we characterize two nuclear effectors of the rice blast pathogen Magnaporthe oryzae. Both nuclear effectors are secreted via the biotrophic interfacial complex, translocated into the nuclei of initially penetrated and surrounding cells, and reprogram the expression of immunity-associated genes by binding on effector binding elements in rice. Their expression in transgenic rice causes ambivalent immunity: increased susceptibility to M. oryzae and Xanthomonas oryzae pv. oryzae, hemibiotrophic pathogens, but enhanced resistance to Cochliobolus miyabeanus, a necrotrophic pathogen. Our findings help remedy a significant knowledge deficiency in the mechanism of $M$. oryzae-rice interactions and underscore how effector-mediated manipulation of plant immunity by one pathogen may also affect the disease severity by other pathogens.

\footnotetext{
${ }^{1}$ Department of Agricultural Biotechnology, Seoul National University, Seoul 08826, Korea. ${ }^{2}$ Department of Plant Medicine, Sunchon National University, Suncheon 57922, Korea. ${ }^{3}$ Interdisciplinary Program in Agricultural Genomics, Seoul National University, Seoul 08826, Korea. ${ }^{4}$ Department of Biotechnology, Yeungnam University, Gyeongsan 38541, Korea. ${ }^{5}$ Graduate School of Biotechnology and Crop Biotech Institute, Kyung Hee University, Yongin 17104, Korea. ${ }^{6}$ Department of Plant Biology, University of Georgia, Athens, GA 30602, USA. 7 Department of Plant Pathology and Environmental Microbiology, The Pennsylvania State University, University Park, PA 16802, USA. ${ }^{8}$ Center for Fungal Genetic Resources, Plant Immunity Research Center, and Research Institute of Agriculture and Life Sciences, Seoul National University, Seoul 08826, Korea. ${ }^{9}$ Present address: Crop Cultivation and Environment Research Division, National Institute of Crop Science, Rural Development Administration, Suwon 16613, Korea. ${ }^{10}$ Present address: Heinrich-Heine University Düsseldorf, Institute for Microbiology, Cluster of Excellence on Plant Sciences, Düsseldorf 40204, Germany. ${ }^{11}$ Present address: Smart Farm Research Center,

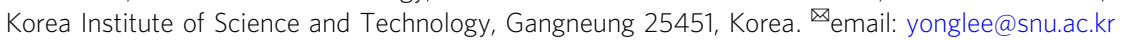


$\mathrm{P}$ lants employ two-layered immune systems for protection from diverse groups of pathogens. Cell surface pattern recognition receptors (PRRs) detect conserved pathogenassociated molecular patterns (PAMPs) to trigger PAMPtriggered immunity $(\mathrm{PTI})^{1}$. Effector-triggered immunity (ETI) gets activated when specific host resistance proteins recognize matching pathogen effectors directly or indirectly. Generally, while PTI confers basal and broad-spectrum resistance, ETI provides race-specific resistance with stronger defense responses, such as the hypersensitive response ${ }^{1}$. Both types of immunity entail large-scale transcriptional reprogramming orchestrated by phytohormones and transcription factors ${ }^{2,3}$.

Pathogens elude or suppress host defense by releasing effectors that collectively function to manipulate pathogen surveillance systems, physiology, and immune responses. Effectors often target host receptors or defense-signaling components to disrupt the initiation of defense responses ${ }^{4}$. Pathogen effectors typically display a high level of genetic diversity due to arms races between plants and pathogens and can be classified according to where they function. Apoplastic effectors are secreted to the outside of plant cells, while cytoplasmic effectors are translocated into the plant cytoplasm via several mechanisms. Several effectors that function in plant nuclei have been identified in oomycetes and bacterial pathogens. Phytophthora Crinkler (CRN) effectors and bacterial Transcription Activator-Like (TAL) effectors suppress host defense or induce susceptibility by manipulating plant gene expression $^{5,6}$. Compared to research on such bacterial and oomycete effectors, research on fungal nuclear effectors lags. Nuclear effector candidates in fungal pathogens, including Ustilago maydis See1, Puccinia striiformis f.sp. tritici PstGSRE1, and Colletotrichum graminicola CgEP1, have been reported ${ }^{7-9}$. However, more work is needed to confirm nuclear localization during infection or whether they control the expression of immunity-associated genes in hosts.

Rice blast, caused by $M$. oryzae, reduces global rice yield by $10-30 \%$ annually ${ }^{10}$. Besides its economic significance, this pathosystem has been extensively investigated, resulting in highquality genome and transcriptome data for rice and M. oryzae. Additionally, protocols for transformation and gene manipulation of both organisms are robust. $M$. oryzae forms an infection structure called the appressorium to penetrate host cells and develops invasive hyphae (IH) in penetrated cells without causing cell death ${ }^{11}$. Cytoplasmic effectors are secreted into the cytoplasm of invaded rice cells via the biotrophic interfacial complex (BIC) ${ }^{11}$. Its apoplastic effectors are secreted into the extra-invasive hyphal membrane (EIHM) compartment ${ }^{11}$.

In this study, we identify and characterize two nuclear effectors of Magnaporthe oryzae, named as MoHTR1 and MoHTR2 for M. oryzae Host Transcription Reprogramming 1 and 2, respectively. Both MoHTR1 and MoHTR2 seem to function as transcription repressors that reprogram the transcription of large numbers of immunity-associated genes in rice. We also identify effector binding elements and uncover candidate target genes of these nuclear effectors. Interestingly, transgenic expression of MoHTR1 and MoHTR2 in rice affects susceptibility to not only $M$. oryzae but also Xanthomonas oryzae pv. oryzae, a hemibiotrophic bacterial pathogen, and Cochliobolus miyabeanus, a necrotrophic fungal pathogen, in a manner that depends on their mode of infection. Our findings suggest that these nuclear effectors modulate disease susceptibility of rice via transcriptional reprogramming of immunity-associated genes.

\section{Results}

Identification of nuclear effectors of $M$. oryzae. We identified nuclear effectors based on the presence of both a secretion signal and a DNA-binding domain. In total, 1895 M. oryzae proteins that lack a glycosylphosphatidylinositol (GPI) anchor, an endoplasmic reticulum (ER) retention signal, and transmembrane helix(es) were curated as secreted proteins in Fungal Secretome Database (FSD; http://fsd.snu.ac.kr) ${ }^{12}$. Among these, nuclear effector candidates that likely participate in transcriptional regulation were identified using Fungal Transcription Factor Database (FTFD; http://ftfd.snu.ac.kr) ${ }^{13}$. Twenty proteins carry a DNA-binding domain, with $50 \%$ of them possessing the $\mathrm{C}_{2} \mathrm{H}_{2}$ zinc finger domain (IPR007087). The remaining proteins carry one of the following domains: high mobility group superfamily (IPR009071), Myb (IPR001005), homeodomain-like (IPR009057), $\mathrm{Zn}_{2} \mathrm{Cys}_{6}$ transcription factor (IPR007219), TFIIS zinc finger (IPR012164), and nucleic acid-binding OB fold (IPR008994). They were named as MoHTR1-20 for M. oryzae Host Transcription Reprogramming 1-20 (Supplementary Table 1). We used WoLF-PSORT ${ }^{14}$, NLStradamus ${ }^{15}$, and cNLS mapper ${ }^{16}$ to check the presence of a potential nuclear localization signal (NLS) in MoHTR1-20. Sixteen proteins were predicted to carry one or more NLS by at least one program (Supplementary Table 1).

Expression patterns and homolog distribution and structural features of MoHTRs. We analyzed how the MoHTR genes are expressed during vegetative growth and in infected rice sheaths at the pre-penetration (18 hours post inoculation, hpi), biotrophic (27 and $36 \mathrm{hpi}$ ), transition (45 hpi), and necrotrophic (72 hpi) stages using available RNA-seq data ${ }^{17}$. Eleven genes were highly expressed during the biotrophic or transition stage, with their $\log _{2}(\mathrm{FPKM}+1)$ being $\geq 4$. Among these, eight genes were considered infection-specific because their transcripts were undetectable or very low in vegetative mycelia (Supplementary Fig. 1).

We used BLASTMatrix, a BLAST tool designed to search multiple genomes simultaneously, in Comparative Fungal Genomics Platform 2.0 (CFGP 2.0) ${ }^{18}$ to search their homologs in diverse species. Genes homologous to some MoHTR genes were present in 97 fungi, 5 oomycetes, 2 plants, and 3 animals. Homologs of MoHTR11, MoHTR13, MoHTR14, MoHTR15, and MoHTR19 are present in most fungal and oomycete species analyzed. Homologs of MoHTR12, MoHTR16, MoHTR18, and MoHTR20 are present in subclades of Ascomycota and Basidiomycota but mostly absent among oomycetes. The remaining 11 genes appear $M$. oryzae-specific. Distribution patterns of all MoHTR genes at the population level were also analyzed using the genomes of $39 \mathrm{M}$. oryzae isolates. Fifteen genes were present in more than 36 isolates.

Two M. oryzae-specific effectors, MoHTR1 and MoHTR2, were selected for further analyses because they were specifically expressed during infection (Supplementary Fig. 1) and accumulated at BIC. We compared their structural features with Drosophila melanogaster GAGA, a well-characterized $\mathrm{C}_{2} \mathrm{H}_{2}$ zinc-finger transcription factor, to infer how MoHTR1 and MoHTR2 interact with DNA. The binding of GAGA to target gene promoters requires a single $\mathrm{C}_{2} \mathrm{H}_{2}$ zinc finger domain and flanking basic amino acid-rich regions ${ }^{19}$. The structure of the $\mathrm{C}_{2} \mathrm{H}_{2}$ zinc finger domain in MoHTR1 and MoHTR2 was predicted via ab initio modeling (Phyre2; http://www.sbg.bio.ic. ac.uk/ phyre 2$)^{20}$. The corresponding domain of $D$. melanogaster GAGA was superimposed to those of MoHTR1 and MoHTR2 using SuperPose version 1.0 (http://wishart.biology.ualberta.ca/ SuperPose/ $)^{21}$. The root mean square deviation (RMSD) between MoHTR1 and GAGA was $2.41 \AA$ and that between GAGA and MoHTR2 was $1.95 \AA$. Similar to GAGA, basic amino acids are present at both sides of the zinc finger domain of MoHTR1 and MoHTR2, suggesting their DNA binding. 
a
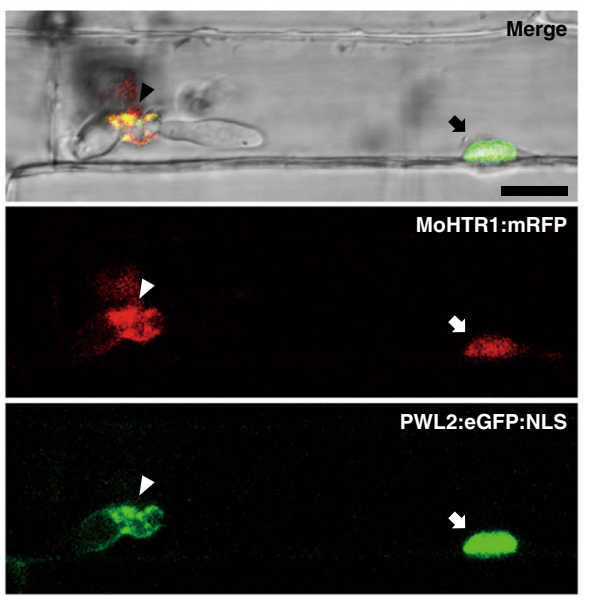

b

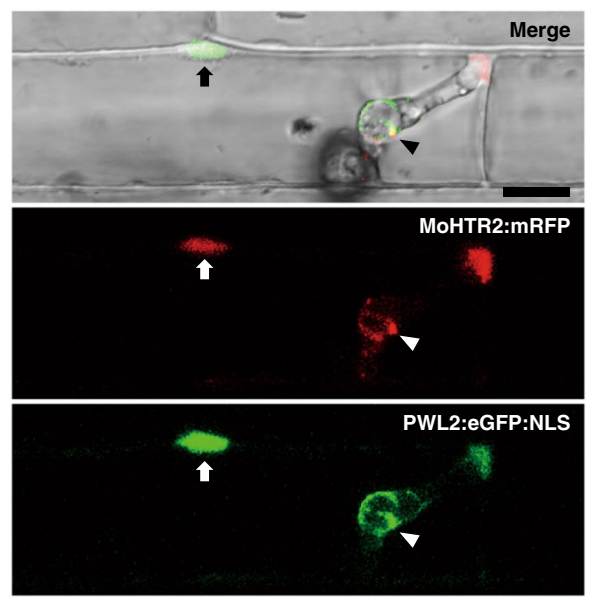

Fig. 1 Localization of MoHTR1 and MoHTR2 in rice nuclei. Differential interference contrast (DIC) and confocal images of rice sheath cells infected by $M$. oryzae at 30 hours post-inoculation (hpi). The fluorescent images shown were chosen from confocal optical z-sections taken at 1- $\mu$ m intervals. Both a

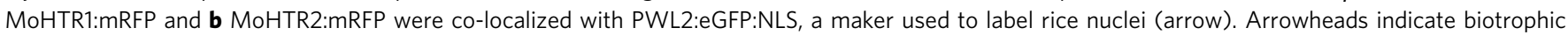

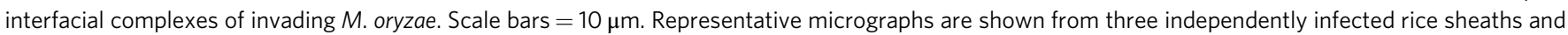
additional representative data are provided as a Source Data file.

In planta localization of MoHTR1 and MoHTR2. To determine where MoHTR1 and MoHTR2 localize during rice infection, fungal transformants expressing these proteins fused to a monomeric RFP (MoHTR:mRFP) under their native promoters were generated. Rice sheaths inoculated with individual transformants were imaged at $30 \mathrm{hpi}$. Consistent with their transcriptional induction during the biotrophic stage, both MoHTR1:mRFP and MoHTR2:mRFP accumulated in BICs and rice nuclei (Fig. 1). Rice nuclei were marked by expressing PWL2, a known cytoplasmic effector of $M$. oryzae ${ }^{11}$, fused to eGFP:NLS (eGFP linked to a NLS of SV40 large T antigen). MoHTR1:mRFP and MoHTR2:mRFP were detected in rice nuclei in $77 \%(n=43)$ and $89 \%(n=37)$ of the infection sites observed, respectively. When fungal hyphae moved into the cells adjacent to initially penetrated cells, both proteins were also detected in their nuclei (Supplementary Fig. 2).

Penetration of $M$. oryzae results in the invagination of the rice plasma membrane. Because the rice plasma membrane remains intact during the biotrophic stage of infection ${ }^{22}$, pathogen effectors must infiltrate the membrane to translocate into rice cells. BAS4 is an apoplastic effector of M. oryzae, and its secretion signal peptide (BAS4sp) linked to a fluorescent protein has been used as a marker for checking the integrity of the rice membrane ${ }^{11}$. When rice sheaths were inoculated with fungal transformants expressing both BAS4sp:eGFP and each MoHTR: mRFP, BAS4sp:eGFP was retained in the apoplast whereas MoHTR1:mRFP and MoHTR2:mRFP were detected in rice nuclei (Fig. 2a, b). M. oryzae cytoplasmic effectors have been shown to move to as-yet-uninvaded rice cells through the plasmodesmata $^{11}$. Similarly, MoHTR1:mRFP and MoHTR2:mRFP were also detected in nuclei of uninvaded rice cells (Fig. $2 \mathrm{c}, \mathrm{d}$ ). The MoHTR1 and MoHTR2 genes that lack the secretion signal peptide (MoHTR1- $\Delta$ sp and MoHTR2- $\Delta$ sp) were fused to $e G F P$ to check their subcellular location in rice protoplasts. Consistent with their nuclear localization in infected rice sheath cells, MoHTR1- $\Delta$ sp and MoHTR2- $\Delta$ sp were mostly located in rice nuclei (Supplementary Fig. 3). Rice nuclei were marked using $\mathrm{ABF} 1$, a rice transcription factor ${ }^{23}$. We also determined whether both MoHTR- $\Delta$ sp proteins localize in fungal nuclei. MoHTR1$\Delta$ sp:mRFP was detected in the cytoplasm, and MoHTR2- $\Delta$ sp: mRFP appeared as speckles and did not accumulate in fungal nuclei (Supplementary Fig. 3).
Identification of effector binding elements for MoHTR1 and MoHTR2. The presence of a $\mathrm{C}_{2} \mathrm{H}_{2}$ zinc finger domain in MoHTR1 and MoHTR2 and their accumulation in rice nuclei led to the hypothesis that they regulate the transcription of rice genes by binding to target gene promoters. We identified candidate binding sequences using a quadruple 9 -mer-based protein binding microarray (PBM) ${ }^{24}$ (Fig. 3a). This approach allowed us to quantify the binding affinity of DsRed-linked MoHTR1 and MoHTR2 to all possible 9-mer DNA sequence probes based on the fluorescence intensity from individual probes on the chip. The rank-ordered signal distribution exhibited a steep rightward slope, indicating that these proteins strongly bind to only a small number of DNA probes. Each 8-mer sequence occurs 36 times in this 9-mer-format DNA microarray. We sorted the median signal intensity for all possible 8-mers to identify effector binding elements (EBEs) (Supplementary Table 2). The highest median intensity was found at CAATCTTC for MoHTR1 and CCACCTCC for MoHTR2. We compared the average signal intensities from EBE variants with a single-nucleotide variation to determine which nucleotide(s) are crucial for binding. All nucleotide changes in the MoHTR1 EBE reduced the signal intensity at varying degrees. The second and third nucleotides appeared most important for MoHTR1 binding. For MoHTR2, all changes except the third position decreased the signal intensity (Fig. 3b).

Identification of the rice genes targeted by MoHTR1 and MoHTR2. We mined the rice genome to identify the candidate genes targeted by MoHTR1 and MoHTR2 based on the presence of $\mathrm{EBE}$ in their promoter region (defined as 1-kb upstream sequences from the coding region) and differential expression during $M$. oryzae infection. We identified 226 (116 up- and 110 down-regulated) and 650 (274 up- and 376 down-regulated) genes that met both criteria as potential targets for MoHTR1 and MoHTR2, respectively (Supplementary Tables 3 and 4). Eleven genes appeared to be common targets of both effectors (Supplementary Table 5). Gene ontology (GO) enrichment analysis was performed using RiceNetDB ${ }^{25}$ to classify their functions. Metabolism-related and stress-responsive terms are commonly enriched among the candidates targeted by MoHTR1 and 
a
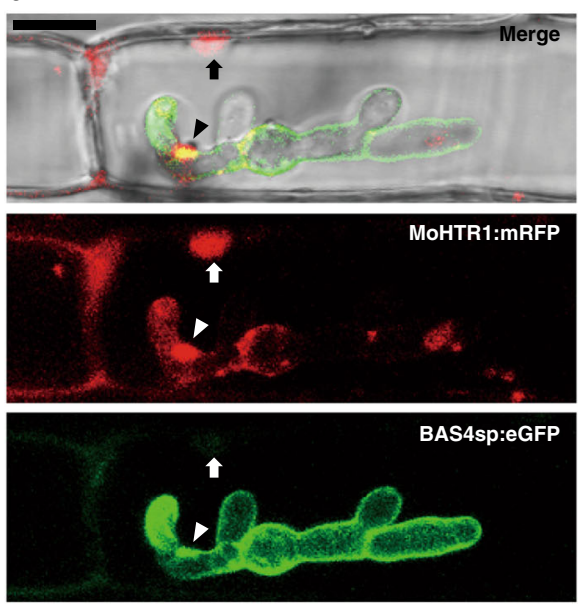

C
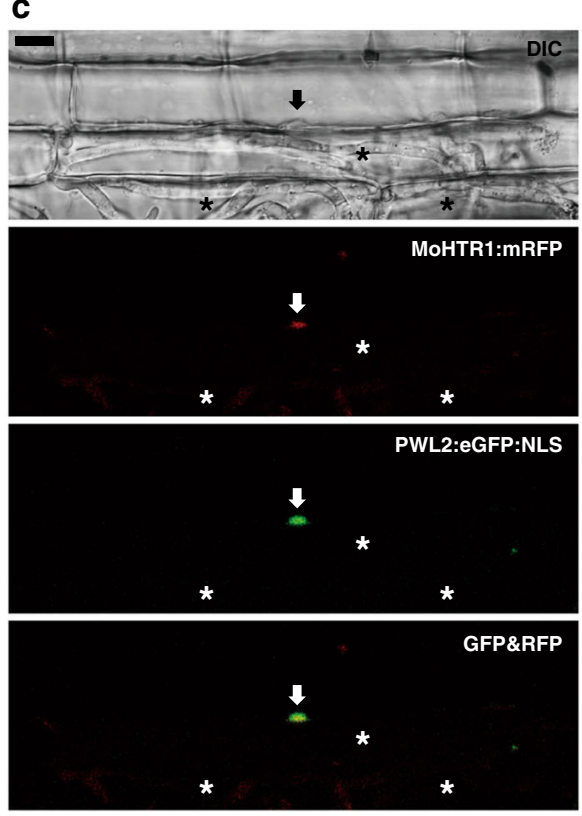

b

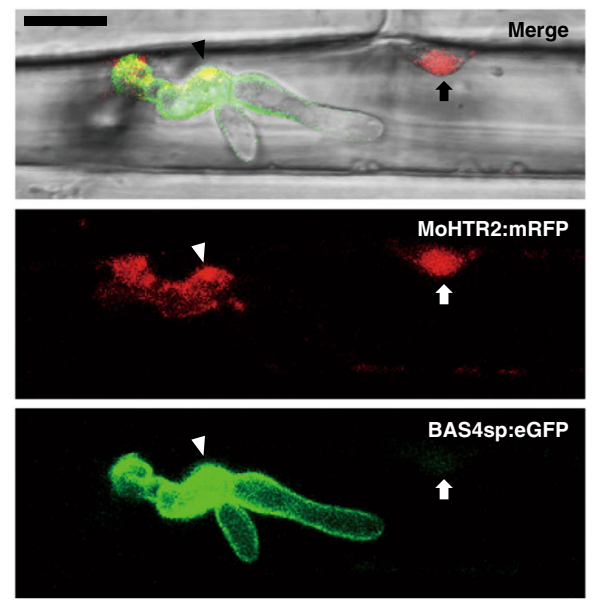

d

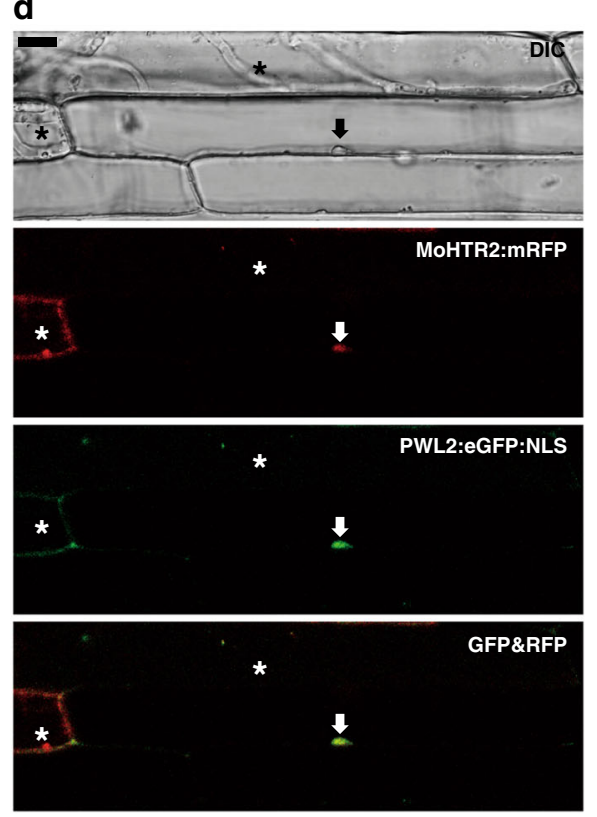

Fig. 2 Translocation of MoHTR1 and MoHTR2 into rice cell and migration into rice nuclei. The MoHTR1:mRFP and MoHTR2:mRFP expressed under control of their native promoter were observed at an early infection stage (30 hpi). BAS4sp:eGFP is a marker for intact extra-invasive hyphal membrane. a MoHTR1:mRFP and $\mathbf{b}$ MoHTR2:mRFP were mainly localized in BICs and the nuclei of invaded rice leaf sheath cells. The images shown were chosen from confocal optical z-sections taken at 1- $\mu \mathrm{m}$ intervals. At 48 hpi, c MoHTR1:mRFP and $\mathbf{d}$ MoHTR2:mRFP were also detected in the nuclei of some uninfected cells. Arrowheads indicate BICs, and arrows indicate rice nuclei. Asterisks indicate rice cells invaded by fungal hyphae. Scale bars $=10 \mu \mathrm{m}$. Representative micrographs are shown from three independently infected rice sheaths and additional representative data are provided as a Source Data file.

MoHTR2 (Supplementary Tables 6 and 7). Forty and 75 potential targets for MoHTR1 and MoHTR2, respectively, were associated with the term "Response to stress (GO: 0006950)", which also includes disease resistance genes (Supplementary Tables 6 and 7). We also found previously characterized immunity-associated genes, including OsMYB4 for MoHTR1 and OsHPL2 and OsWRKY45 for MoHTR2 26-28. We determined whether MoHTR1 and MoHTR2 bind to their target gene promoters in vivo via yeast one-hybrid analysis. Both MoHTR- $\Delta$ sps fused to the GAL4 activation domain (AD) were expressed using the yeast $A D H 1$ promoter. The 38-bp promoter fragments from three target genes, which included EBE, were used. Both AD:MoHTR1$\Delta \mathrm{sp}$ and AD:MoHTR2- $\Delta \mathrm{sp}$ activated the reporter gene expression (Fig. 3c), suggesting that MoHTR1 and MoHTR2 bind to these promoters. No reporter gene expression was detected in the negative control.
We also performed a luciferase (LUC)-based assay by transiently expressing these MoHTR effectors in rice protoplasts to confirm their binding to selected target gene promoters in planta. The LUC reporter gene was cloned under the promoter of OsMYB4 or OsWRKY45. The luminescence signal increased only when each MoHTR effector linked to the TAL activation domain was expressed in rice protoplasts (Fig. 3e), supporting that the MoHTR effectors interact with the target gene promoters.

MoHTR1 and MoHTR2 regulate transcription in rice protoplasts. We transiently expressed MoHTR 1 and MoHTR 2 in rice protoplasts to determine how they regulate rice gene expression. We designed to express the LUC gene under the control of target promoters of MoHTR effectors (OsMYB4 for MoHTR1 and OsWRKY45 for MoHTR2) to investigate the nature of 
a

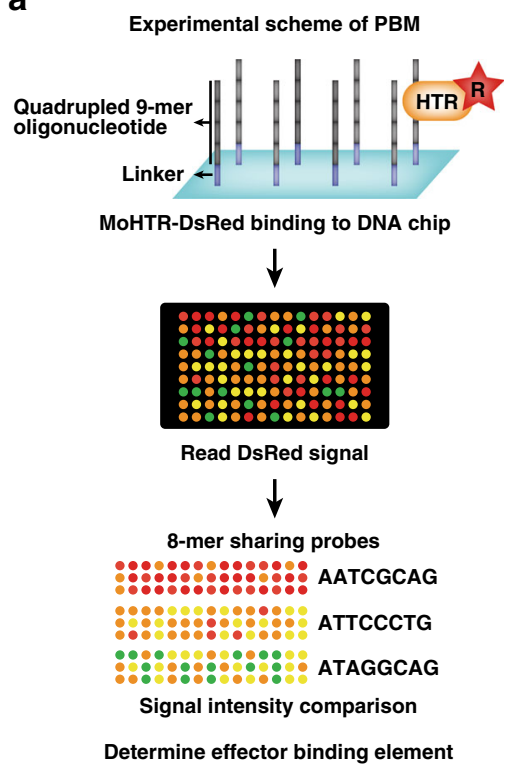

b

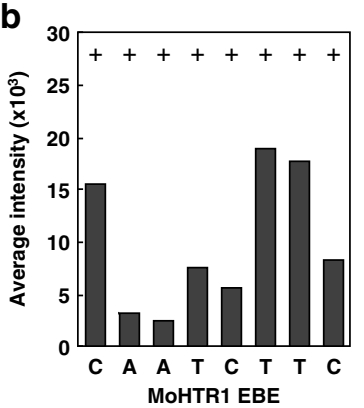

C
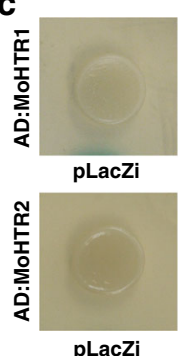

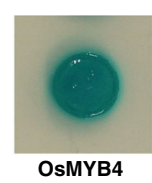

OsMYB4

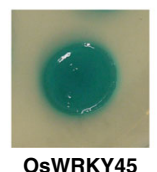

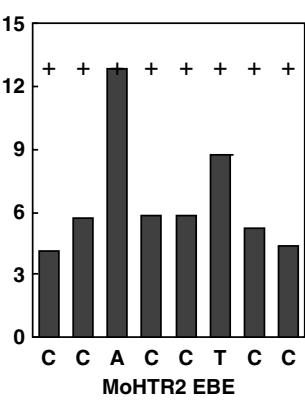

d
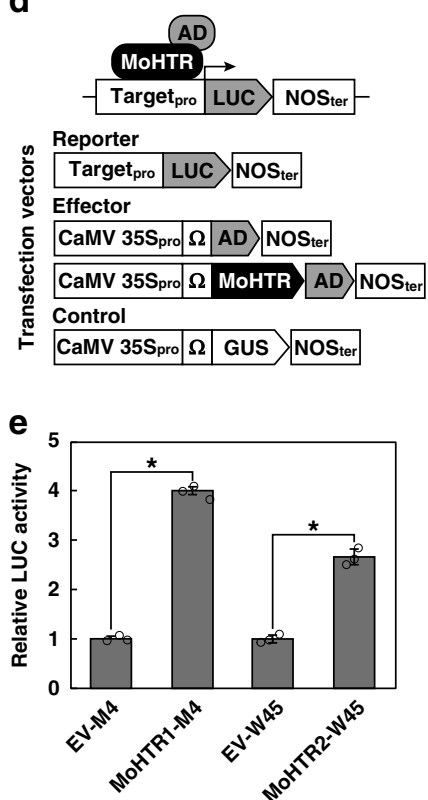

Fig. 3 Identification of effector binding elements targeted by MoHTR1 and MoHTR2. a Effector binding elements (EBEs) targeted by MoHTR1 and MoHTR2 were identified using a protein binding microarray. Each MoHTR- $\Delta$ sp:DsRed (R) protein produced using E. coli was applied to DNA chip containing quadrupled 9-mer nucleotides. The EBE for each protein was identified based on the fluorescent intensity from the group of elements containing the same 8-mer nucleotide. $\mathbf{b}$ Effect of a single nucleotide mismatch in each EBE on the binding affinity to MoHTR. The line denoted using + indicates the signal intensity from EBE without variation. Bars represent the average signal intensity from individual EBE variants with each carrying a different singlenucleotide mismatch (noted in $x$-axis). c Schematic representation of a yeast one-hybrid assay used for detecting MoHTR-EBE interaction and resulting data. NLS:AD:MoHTR- $\Delta$ sp was expressed under the ADH1 promoter. NLS, a nuclear localization signal from the Simian Virus 40 large T antigen; AD, GAL4 activation domain. The reporter, $\beta$-galactosidase (GAL), was placed under the recombinant promoter comprised of an EBE-centered 38-bp fragment and a minimal cytochrome $\mathrm{C} 1$ promoter (CYC1). GAL expression was activated when NLS:AD:MoHTR- $\Delta$ sp binds to its EBE in the promoter. pLacZi was used as a negative control. d Validation for binding of MoHTRs and target promoters in planta. LUC was regulated by the corresponding gene promoter. Activation domain of TAL effector (AD) was linked to MoHTR- $\Delta$ sp and expressed under the $35 \mathrm{~S}$ CaMV promoter. e Relative LUC activities in rice protoplasts transfected with AD-linked MoHTR- $\Delta$ sp were compared with those transfected with the EV and corresponding reporter vector (M4: OsMYB4 promoter, W45: OsWRKY45 promoter). $n=3$ independently transfected protoplasts; mean \pm standard deviation (SD); ${ }^{\star} P<0.01$, two-sided Student's $t$-tests. Source data underlying Fig. $3 e$ are provided as a Source Data file.

transcriptional reprogramming by these effectors (Fig. 4a). Each MoHTR- $\Delta$ sp suppressed the LUC expression (Fig. 4b), suggesting that both MoHTR1 and MoHTR2 function as a transcriptional repressor. We used the LUC gene under the control of GAL4 upstream activation sequence (UAS)-linked $35 \mathrm{~S}$ minimal promoter $\left(35 \mathrm{~S}_{\text {mini }}\right.$ promoter) to induce basal level expression. We also expressed GAL4 DNA binding domain (GBD)-linked MoHTR- $\Delta$ sp for binding to UAS:35S $\mathrm{S}_{\text {mini }}$ promoter (Supplementary Fig. 4). The basal expression of $L U C$ under the UAS: $35 S_{\text {mini }}$ promoter was not changed by GBD:MoHTR- $\Delta$ sp expression (Supplementary Fig. 4), suggesting that MoHTR1 and MoHTR2 may not have an intrinsic repressor activity and recruit unknown repressors(s).

MoHTR1 and MoHTR2 reprogram the expression of many immunity-associated genes in rice. To investigate how MoHTR1 and MoHTR2 modulate the expression of target genes during infection, we generated deletion mutants of MoHTR1 and MoHTR2 in M. oryzae KJ201. We compared transcript levels of target genes in $\triangle M o h t r$-infected rice plants with those in control plants (infected with KJ201). The transcript level of OsMYB4 in $\Delta$ Mohtr1-infected rice increased 1.6-fold over that of the control. In $\triangle$ Mohtr2-infected plants, the expression of OsHPL2 and OsWRKY45 was induced by $2.6-$ and 3.1 -folds, respectively (Fig. 4c). These results are consistent with the model that MOHTR1 and MoHTR2 encode transcription repressors for regulating expression of their target genes in rice.
We also generated transgenic lines of the rice cultivar Nakdong that express the MoHTR1- $\triangle$ sp and MoHTR2- $\Delta$ sp individually under the control of the CaMV $35 \mathrm{~S}$ promoter. Transgenic lines that highly expressed MoHTR1 (MoHTR1-OX) and MoHTR2 (MoHTR2-OX) were chosen. Transcript levels of MoHTR1 and MoHTR2 in these MoHTR-OX lines were up to 6.5- and 9.6-folds higher than that of rice Actin. We analyzed how target genes in two independent MoHTR-OX lines for each effector were affected compared to their expression in Nakdong. The expression of OsMYB4 decreased in the MoHTR1-OX lines (up to 0.2-fold). In the MoHTR2-OX lines, both OsHPL2 and OsWRKY45 were downregulated (up to 0.3- and 0.6-folds, respectively) (Fig. 4d). These results provide further evidence supporting the role of MoHTR1 and MoHTR2 as transcription repressors in rice.

MoHTR1 and MoHTR2 also affected the expression of other immunity-associated genes that were not directly targeted by these effectors. Expression of several immunity-associated genes such as pathogenesis-related $(P R)$ and hormone signaling genes increased when rice was infected with $\Delta$ Mohtr1 and $\Delta$ Mohtr2 (Supplementary Fig. 5). Moreover, the expression of PR5, PR10a, and salicylic acid-related genes was downregulated in the MoHTR-OX lines (Supplementary Fig. 5). To identify rice genes affected by these nuclear effectors genome-wide, we performed RNA-seq analyses of MoHTR1-OX and MoHTR2-OX lines. More than 1,000 differentially expressed genes (DEGs) were found in both transgenic lines. A GO enrichment analysis revealed that the genes associated with "response to biotic stimulus (GO:0009607)" 
a
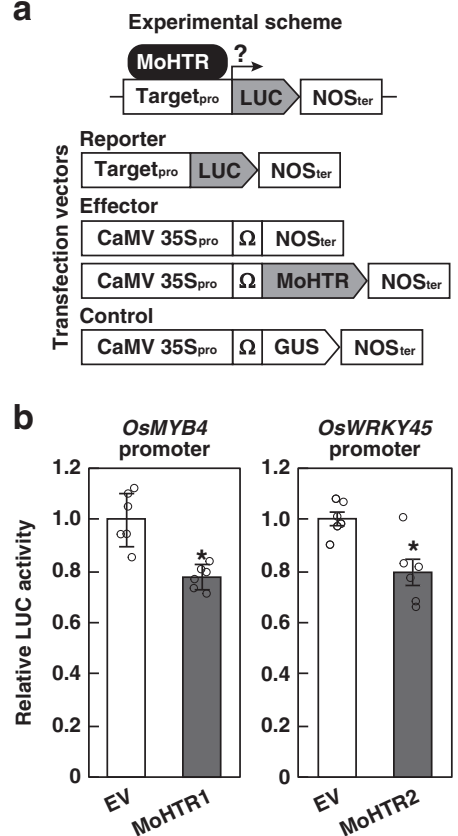

C

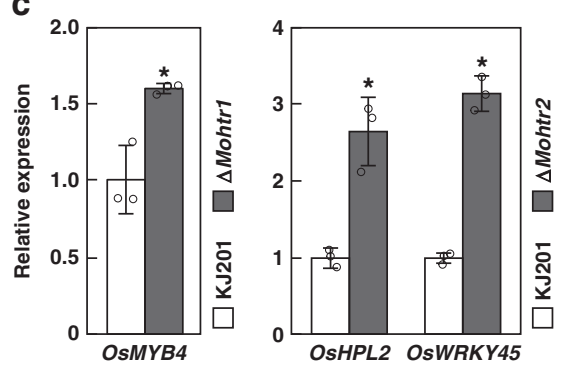

d
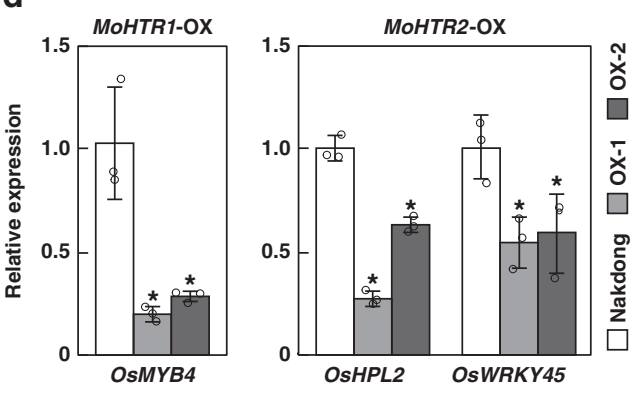

Fig. 4 Transcriptional regulation of rice genes targeted by MoHTR1 and MoHTR2. a Features of the constructs used to determine how MoHTR1 and MoHTR2 affect the expression of LUC under each rice gene promoter tested in rice protoplasts. MoHTR1- $\Delta$ sp and MoHTR2- $\Delta$ sp were expressed using the CaMV 355 promoter and the translation enhance sequence $\Omega$. b Relative LUC activities in protoplasts transfected with MoHTR1- $\Delta$ sp and MoHTR2- $\Delta$ sp were compared with those transfected with the empty vector (EV). $n=6$ independently transfected protoplasts; mean \pm SD; ${ }^{\star} P<0.01$, two-sided Student's t-tests. Three biological replicates produced similar results. $\mathbf{c}$ Expression patterns of the selected target genes in rice individually infected with $\Delta$ MoHTR1 and $\triangle$ MoHTR2 were compared with those in rice infected with KJ201. Mean \pm SD, three independent experiments; ${ }^{\star} P<0.01$, two-sided Student's $t$-tests. d Expression levels of individual target genes in MoHTR1-OX and MoHTR2-OX were compared with those in Nakdong (wild type) using qRT-PCR. Mean \pm $\mathrm{SD}$, three independent experiments; ${ }^{\star} P<0.05$, one-way analysis of variance (ANOVA) with Tukey's honest significant difference (HSD) test. Detailed information about biological repeat experiments and statistical analysis underlying Fig. $4 b-d$ are described as a Source Data file.

were commonly downregulated in MoHTR1-OX and MoHTR2OX (Supplementary Tables 8 and 9). We found that $64.5 \%$ (240 genes) of the up-regulated genes in MoHTR1-OX were also upregulated in MoHTR2-OX. Similarly, 36.6\% (286 genes) of the down-regulated genes in MoHTR1-OX were also down-regulated in MoHTR2-OX (Supplementary Fig. 6). The percentages of EBEcontaining DEGs in the promoter are 3.4\% (40 genes out of 1,153 DEGs) in MoHTR1-OX and 12.3\% (138 genes out of 1,115 DEGs) in MoHTR2-OX. Some of the not-affected genes contain EBE in the promoter, but the percentages are lower: $3.18 \%$ (1,383 genes) in MoHTR1-OX and 7.44\% (3,237 genes) in MoHTR2-OX.

Effect of MoHTR deletion on fungal development and virulence. We tested if MoHTR1 and MoHTR2 regulate fungal development by observing the vegetative growth, conidiation, conidial germination, and appressorium formation of $\Delta$ Mohtr1 and $\Delta M o h t r 2$. We observed no significant differences between the mutants and KJ201 (Supplementary Table 10). However, the mutants exhibited significantly reduced virulence to rice. The average disease indices caused by $\Delta$ Mohtr 1 and $\Delta M o h t r 2$ were 2.5 and 2.2, respectively, whereas that of KJ201 was 4.6 (Fig. 5). Rice sheaths were also inoculated for virulence assessment. The severity of invasion was quantified by rating the degree of infection progress (from 1 to 4 ) in over 100 infection sites (Supplementary Fig. 7). Invasive hyphae of KJ201 caused types $2-4$ at $66.9 \%$ of the infection sites. However, $\Delta$ Mohtr1 and $\Delta$ Mohtr2 caused types $2-4$ at $23.7 \%$ and $26.5 \%$ of the infection sites, respectively. Complemented strains of $\Delta$ Mohtrl and $\Delta$ Mohtr2 displayed virulence level comparable to that of KJ201 (Supplementary Fig. 7). We also inoculated barley seedlings with these mutants to determine whether the function of these effectors is rice specific. Virulence of $\Delta M o h t r 1$ and $\Delta M o h t r 2$ was significantly reduced (Supplementary Fig. 8), indicating that their function is not rice specific.

Expression of MoHTR1 and MoHTR2 in rice affects susceptibility to multiple pathogens. We investigated whether MoHTR1-OX and MoHTR2-OX lines are more susceptible to $M$. oryzae. In sheaths of wild-type rice plants inoculated with KJ201, only $3.3 \%$ of the infection sites exhibited type 3 invasion at $48 \mathrm{hpi}$. However, in MoHTR1-OX and MoHTR2-OX, 22.4 and 35.2\% of the infection sites, respectively, displayed type 3 or 4 at $48 \mathrm{hpi}$ (Fig. 6a). We also determined whether the expression of MoHTR1 or MoHTR2 in rice affects susceptibility to other pathogens by infecting MoHTR1-OX and MoHTR2-OX lines with Xanthomonas oryzae pv. oryzae (Xoo), a hemibiotrophic bacterial pathogen that causes rice bacterial leaf blight, and C. miyabeanus, a necrotrophic fungal pathogen that causes rice brown spot. Both MoHTR1-OX and MoHTR2-OX infected with Xoo displayed more severe symptoms than wild-type plants (Fig. 6b). In contrast, necrotic lesions on both transgenic lines infected with $C$. miyabeanus were much smaller than those on wild type (Fig. 6c), suggesting that transcriptional reprogramming in rice by MoHTR1 and MoHTR2 alter rice resistance/susceptibility depending on the pathogen's mode of infection.

\section{Discussion}

Plant nuclei are the control hub for the immune system against diverse pathogens; signals generated upon the recognition of pathogens are conveyed to plant nuclei in which the signals are decoded into a cascade of molecular and cellular changes needed to execute appropriate defense responses ${ }^{29}$. Here, we show that 


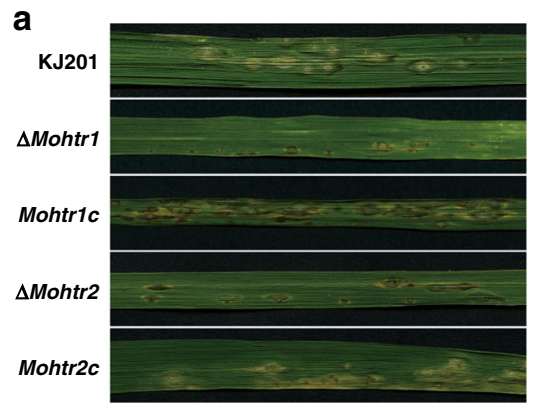

b

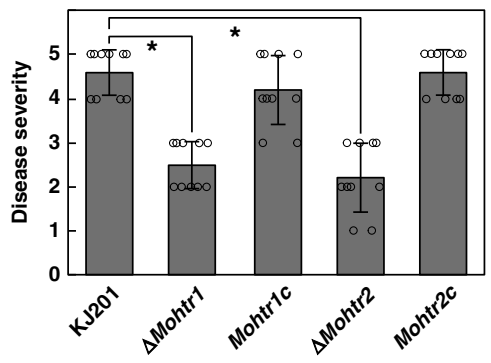

Fig. 5 The requirement of MoHTR1 and MoHTR2 for full virulence. 4week-old Nakdong rice seedlings were sprayed with conidial suspensions $\left(5 \times 10^{4} \mathrm{~mL}^{-1}\right)$ of KJ201 (wild type), two MoHTR knockout mutants, and complemented mutant strains. a Representative infected leaves were collected at $7 \mathrm{dpi}$, and $\mathbf{b}$ quantified data are shown. $n=10$ independently inoculated plants; mean $\pm S D$; ${ }^{\star} P<0.05$, one-way ANOVA with Tukey's HSD test. Three independent infection assays produced similar results. Detailed information about biological repeat experiments and statistical analysis underlying Fig. $5 b$ are provided as a Source Data file.

M. oryzae delivers two nuclear effectors into rice nuclei to reprogram the expression of many immunity-associated genes. Besides enhancing susceptibility against $M$. oryzae, the reprogramming in gene expression by these effectors also significantly affected rice defense responses to other pathogens (Fig. 6), suggesting that the genes and processes modulated by the effectors are involved in defense against diverse pathogens. The discovery and functional validation of these novel nuclear effectors were accomplished via a series of experiments: (i) genome mining to identify potential nuclear effectors based on their sequence features associated with protein secretion and DNA binding; (ii) RNA-seq and protein structural modeling analyses to evaluate if their expression is induced during infection and characterize the structure of their DNA-binding domain, respectively; (iii) live-cell imaging of fluorescently-tagged MoHTRs to demonstrate their secretion and translocation into rice nuclei during infection; (iv) protein binding microarray and yeast one-hybrid assays to identify the effector binding element for each MoHTR; (v) both transient and stable expressions of each MoHTR in rice to demonstrate their regulation of the transcription of immunityassociated genes; and (vi) evaluation of how transgenic rice lines expressing each MoHTR respond to bacterial and fungal pathogens that employ different modes of infection.

Plant pathogens deliver cytoplasmic effectors into host cells via several specialized systems. Bacterial pathogens directly inject their effectors using the type III secretion system ${ }^{30}$, and oomycete pathogens deliver effectors by a system that involves host targeting signals ${ }^{31}$. Fungal pathogens have been shown to secrete cytoplasmic effectors via multivesicular bodies or focal membrane-rich structure ${ }^{32,33}$. Cytoplasmic effectors of $M$. oryzae are secreted to the BIC via an unconventional secretory pathway, involving exocyst and SNARE proteins, and translocated into the a
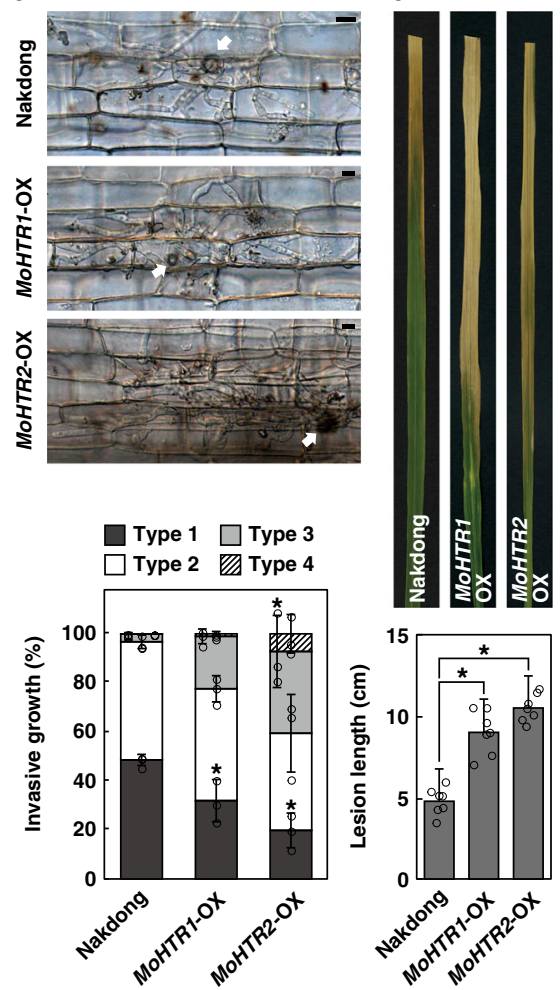

C
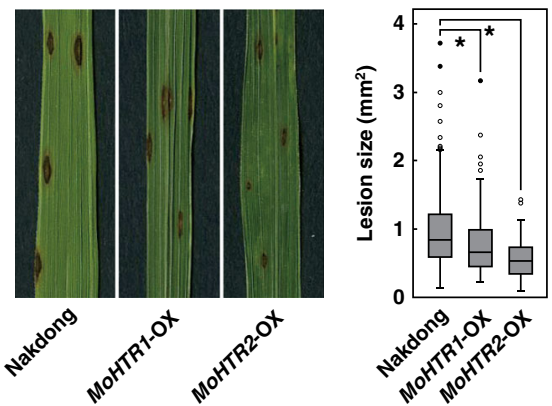

Fig. 6 Expression of MoHTR1 and MoHTR2 in rice affects disease susceptibility to multiple pathogens. a Invasive growth of KJ201 in sheath cells of Nakdong, MoHTRT-OX, and MoHTR2-OX at 48 hpi was observed under light microscopy and rated (from type 1 to type 4 as described in the Methods). The arrow indicates the appressorium on initially penetrated cells. Each bar represents the proportion of individual infection types. $n=3$ independent experiments; mean $\pm S D$; ${ }^{\star} P<0.05$, one-way ANOVA (in randomized block design) with Tukey's HSD test. Scale bars $=10 \mu \mathrm{m}$. b Representative disease symptoms caused by $X$. oryzae pv. oryzae (Xoo) at $7 \mathrm{dpi}$ and lesion length. $n=7$ independently inoculated plants; mean $\pm S D$; ${ }^{*} P<0.05$, one-way ANOVA with Tukey's HSD test. c Representative disease symptoms caused by C. miyabeanus at $7 \mathrm{dpi}$ and lesion size distribution. $n=349$ for Nakdong, $n=143$ for MoHTR1OX, $n=95$ for MoHTR2-OX; ${ }^{\star} P<0.05$, Kruskal-Wallis test with pairwise comparison. Boxes represent median, first, and third quartile. Whiskers show the highest and lowest values that are within 1.5 interquartile range. Three independent experiments produced similar results, and data from one experiment are presented. Detailed information about biological repeat experiments and statistical analysis are provided as a Source Data file.

host cytoplasm ${ }^{34}$. Live-cell imaging of fluorescently-tagged MoHTR1 and MoHTR2 clearly showed that both effectors were secreted through BIC to enter rice cells and subsequently migrated into rice nuclei (Figs. 1 and 2). These effectors contain predicted NLSs that are distinct from those present in known 
nuclear effectors. While nuclear localization of TAL and SP7 effectors was observed in multiple organisms ${ }^{35,36}$, MoHTR1- $\Delta$ sp: RFP and MoHTR2- $\Delta$ sp:RFP moved to rice nuclei but not $M$. oryzae nuclei (Supplementary Fig. 3), suggesting that MoHTR1 and MoHTR2 may require host factors for moving into the nucleus. However, the mechanism underpinning their nuclear localization still remains to be characterized. Similar to an earlier report showing the movement of PWL2:tdTomato, a fluorescent protein-tagged $M$. oryzae cytoplasmic effector, through plasmodesmata $(\mathrm{PD})^{11}$, MoHTRs also appear to translocate into the nuclei of neighboring cells, presumably via $\mathrm{PD}$, before the hyphal invasion.

We identified EBEs for MoHTR1 (CAATCTTC) and MoHTR2 (CCACCTCC) in the rice genome (Fig. 3). Each nucleotide substitution in EBE significantly reduced DNA binding affinities, consistent with their sequence-specific binding. Although we focused on characterizing two EBEs, they are unlikely to be the only target sequences for MoHTRs. Other potential EBE candidates still exhibited strong binding affinities (Supplementary Table 2). Since it is not yet clear if the binding affinity correlates with the degree of suppression in gene expression, the possibility that these effectors also target those genes with other EBEs warrants further studies. Our approach to identifying rice genes potentially targeted by these effectors was not only based on the presence of $\mathrm{EBE}$ in the promoter region but also differential expression during $M$. oryzae infection. Using these criteria, we could efficiently identify candidate target genes associated with rice immunity, including previously reported defense/resistancerelated genes ${ }^{26-28}$.

Transcripts of the genes targeted by MoHTR1 and MoHTR2 increased in rice plants infected by $\Delta$ Mohtr1 and $\Delta$ Mohtr2, while their expression was reduced in MoHTR-OX lines (Fig. 4). These results support the hypothesis that MoHTR1 and MoHTR2 function as repressors of target gene expression. A transactivation assay in rice protoplasts suggests that MoHTR1 and MoHTR2 do not have an intrinsic repressor activity (Supplementary Fig. 4). One possibility is that these effectors may suppress the expression of their targets by interfering with the binding of rice transcription activators through competition for the same binding sites. It is also possible that they may interact with transcription repressor (s) to exert their effect. In addition to directly repressing specific target genes, MoHTR1 and MoHTR2 also appear to modulate the expression of many other immunity-associated genes, including hormone signaling and basal defense genes, indirectly (Supplementary Fig. 5). These data, together with RNA-seq analysis of MoHTR-OX lines, strongly suggest that MoHTR-mediated transcriptional reprogramming likely causes broad changes in rice immunity. Although whether MoHTR1 and MoHTR2 affect immunity-related gene expression in barley remains unknown, the reduced virulence of $\Delta$ Mohtr1 and $\Delta$ Mohtr2 in barley (Supplementary Fig. 8) suggests that they also reprogram such genes in barley. A comparative gene expression analysis between rice and barley after infecting with $\Delta$ Mohtr1 and $\Delta$ Mohtr2 likely reveals similarities and differences in how these cereals defend against $M$. oryzae.

Consistent with the genome-wide transcriptional reprogramming by MoHTR1 and MoHTR2, the resulting effect on rice immunity went beyond defense against $M$. oryzae. Transgenic expression of MoHTR1 and MoHTR2 in rice increased susceptibility to $M$. oryzae and Xoo, hemibiotrophic pathogens, but enhanced resistance to a necrotrophic pathogen, C. miyabeanus (Fig. 6). Further studies are needed to understand how the overexpression of these two nuclear effectors with disparate binding sites similarly affected rice defense against three different pathogens. However, we can speculate a likely mechanism. Plants rely on common defense machineries against different pathogens rather than employing pathogen-specific machineries because evolving defense systems customized for individual pathogens is not possible. This is why although different pathogens have evolved a wide variety of strategies and effectors to dismantle or suppress host defense, they often target similar genes and processes. Both MoHTR1 and MoHTR2 affected the expression of several immunity-associated genes such as some PR genes and phytohormone signaling genes (Supplementary Fig. 5). In addition, 64.5\% (240 genes) of the up-regulated genes in MoHTR1OX rice were also up-regulated in MoHTR2-OX. Similarly, 36.6\% (286 genes) of the down-regulated genes in MoHTR1-OX rice were also down-regulated in MoHTR2-OX rice (Supplementary Fig. 6). Similar rice gene expression patterns resulting from overexpressing MoHTR1 and MoHTR2 likely underpin similar effects on resistance/susceptibility to multiple pathogens.

The ambivalent immunity to multiple pathogens caused by MoHTRs highlighted a critical consideration in developing disease management strategies through targeted gene manipulations. Considering the likelihood that multiple pathogens simultaneously attack rice in the field, the overall plant health is likely to be determined by which combinations of pathogens invade rice. Genetic manipulations of resistance/susceptibility genes to increase resistance against one pathogen may inadvertently create a double-edged sword to plant health by creating unintended susceptibility to other pathogens. This ambivalent immunity and underlying mechanism should be investigated to understand complex plant-pathogen interactions in nature and apply this understanding to develop durable resistance against multiple pathogens.

Our work demonstrates that $M$. oryzae employs nuclear effectors to reprogram the transcription of immunity-associated genes in rice and that some of them likely function in other plants. Characterization of how these fungal nuclear effectors modulate host immunity will help answer multiple questions crucial for understanding the mechanism of plant-pathogen interactions, such as how fungi evade host defense, which plant genes are needed for defense against specific pathogens, and how such plant genes work together or antagonize in responding to diverse pathogens. This understanding should help guide the development of effective strategies for managing plant diseases of economic or environmental significance.

\begin{abstract}
Methods
Genome mining to identify nuclear effector candidates and identification of their target genes in rice. Nuclear effector candidates were mined from the genome of M. oryzae strain 70-1537 archived in Comparative Fungal Genomics Platform 2.0 (CFGP2.0; http://cfgp.snu.ac.kr) ${ }^{18}$. Secreted proteins and transcription factors of $M$. oryzae were curated in Fungal Secretome Database (FSD; http://fsd. snu.ac.kr) ${ }^{12}$ and Fungal Transcription Factor Database (FTFD; http://ftfd.snu.ac. $\mathrm{kr})^{13}$, respectively. BLASTMatrix, a tool in CFGP2. $0^{18}$, was used to search the homologs of MoHTR effectors in the genomes of diverse species and different $M$ oryzae isolates. We used WoLF-PSORT ${ }^{14}$, NLStradamus ${ }^{15}$, and cNLS mapper ${ }^{16}$, to check the presence of potential nuclear localization signal(s) in MoHTR effectors.

We collected 1-kb upstream sequences from the coding region of all rice genes from the Rice Annotation Project Database (RAP-DB) ${ }^{38}$ and checked the presence of each EBE. Differentially expressed genes (DEGs) at $36 \mathrm{hpi} \mathrm{(biotrophic} \mathrm{stage)}$ were identified using transcriptome data generated from infected rice sheaths ${ }^{17}$ Gene ontology (GO) enrichment analysis was performed using RiceNetDB ${ }^{25}$. Information related to previously characterized genes was collected from the funRiceGenes Database ${ }^{39}$.
\end{abstract}

Assays for the subcellular location of MoHTR proteins. A fragment containing the 1-kb upstream and coding regions of each MoHTR gene was inserted to pFPLRh vector ${ }^{40}$ for MoHTR:mRFP expression. Plasmids containing PWL2pro:PWL2: eGFP:NLS and BAS4pro:BAS4sp:eGFP ${ }^{11}$ were used to label plant nuclei and EIHM compartment, respectively. After inoculating rice sheaths with fungal transformants, the location of each protein was microscopically imaged. pFPL-Rh vectors that express each MoHTR- $\Delta$ sp:mRFP under control of the Fusarium verticillioides EF1 a promoter were used to investigate the localization of individual MoHTRs in fungal cells. Fungal transformants expressing these recombinant proteins were 
generated via PEG-mediated transformation. Their expression was confirmed by microscopically observing the fluorescent signal in the conidia of individual transformants. MoHTR1- $\Delta \mathrm{sp}$ and MoHTR2- $\Delta$ sp were cloned into $\mathrm{pENTR}^{\mathrm{TM}} / \mathrm{D}$ TOPO (Invitrogen) to determine their localization in the rice cytoplasm. After confirming the presence of each insert via sequencing, these constructs were moved to the destination vector p7FGW2 for C-terminal GFP fusion ${ }^{41}$ using the Gateway LR clonase (Invitrogen). The primers used for fungal transformation are listed in Supplementary Table 11. The resulting constructs were introduced into rice mesophyll protoplasts using PEG-calcium mediated transformation method ${ }^{42}$ OsABF1:mRFP was used to label plant nuclei ${ }^{23}$.

Confocal laser scanning microscope LSM710 (Carl Zeiss) with C-Apochromat $40 \times / 1.20 \mathrm{~W}$ Korr M27 water immersion objective was used for imaging. The excitation/emission wavelengths for eGFP and mRFP were $488 / 492-562 \mathrm{~nm}$ and 543/590-725 nm respectively, and the pinhole setting for emission fluorescence was 2 airy units. The Axio Imager A1 microscope (Carl Zeiss) was used to acquire epifluorescence and differential interference contrast (DIC) images. AxioCam HRc camera and Axiovision version 4.8 were used for image acquisition. The light source was HBO 100, a 100-W high-pressure mercury plasma arc-discharge lamp.

Protein binding microarray to identify MoHTR-binding DNA sequences. The DNA microarray used contains 232,145 probes that consist of quadruple 9-mer oligonucleotides and cover all possible 9-mer sequences (Agilent Technology). Tandemly repeated 9 -mers were followed by $5^{\prime}$-CGGAGTCACCTAGTGCAG-3 and a 5-nucleotide thymidine linker to the chip $^{24}$. Each DsRed:6× His-linked MoHTR- $\Delta$ sp was expressed using pET-32a vector (Novagen) in Escherichia coli strain BL21-CodonPlus (Stratagene). Recombinant proteins were purified using TALON metal affinity resins (Clontech). Protein binding to the microarray chip was performed in the reaction solution [ $200 \mathrm{nM}$ protein in PBS, $2 \% \mathrm{BSA}, 50 \mathrm{ng} / \mu \mathrm{l}$ salmon-testes DNA (Sigma) and $50 \mu \mathrm{M}$ zinc acetate ${ }^{24}$. The fluorescent signal resulting from MoHTR- $\Delta$ sp-DNA probe interaction was detected using Genepix 4000B microarray scanner (Molecular Devices). Multiple orders of $k$-mer $(k \leq 9)$ repeatedly appear in the DNA microarray chip because each 9-mer sequence is quadrupled. There are 32,768 combinations of 8 -mer nucleotides that repeatedly appear in 36 different probes. We compared the median intensity of probes containing a particular 8-mer to identify the strongest effector binding element for each effector.

Validation of MoHTR-DNA interaction in vivo. For yeast one-hybrid, the EBEcentered 38 bp promoter fragment from each rice gene was inserted into the minimal promoter of yeast cytochrome $\mathrm{Cl}$ (CYC1) gene in pLacZi (Clontech). The fragments used correspond to the -337 to -299 region from the start codon of OsMYB4 and the -766 to -728 region of OsWRKY45. Because there are two binding sites for OsHPL2: -875 to -837 and -745 to -707 from the start codon, both sequences were used for analysis. The $\beta$-galactosidase reporter was placed under each modified $\mathrm{CYC1}$ promoter containing a MoHTR-target site. Each reporter vector was introduced to yeast strain YM4271 via LiAc-mediated transformation, and transformants were selected on SD-ura medium. A transformant without reporter auto-activity was used as a control strain. Each MoHTR- $\Delta$ sp was inserted into pDEST22 (Invitrogen) to fuse it with the sequence encoding the GAL4 activation domain. The recombinant vectors were introduced to the control strains. Transformants were selected on SD-ura-trp medium and transferred to YPD agar supplemented with $0.004 \% \mathrm{X}$-gal (W/V). Blue staining resulting from DNA-protein interaction was checked after 5 days of incubation.

For an in planta interaction assay, reporter vectors containing the luciferase (LUC) gene under the OsMYB4 and OsWRKY45 promoters were generated. We constructed pADT (for Activation Domain Tag) by inserting a quadrupled activation domain (AD) of the Xoo TAL effector AvrXa10 at the downstream of the gateway cassette in $\mathrm{pH} 2 \mathrm{GW} 7^{41}$. Each $M o H T R-\Delta$ sp was integrated in $\mathrm{pADT}$ using LR clonase (Invitrogen). The CaMV $35 \mathrm{~S}$ promoter was used to express each MoHTR- $\Delta$ sp linked to AD. The control vector that contains the beta-glucuronidase (GUS) gene under the maize ubiquitin promoter was used for internal control (Fig. 3d). The effector-DNA interaction was quantified by measuring luminescence after co-transfecting the effector, reporter, and internal control vectors into rice protoplasts. Normalized luminescence (luminescence/GUS activity) resulting from effector expression was compared with that of the empty vector control.

Transcriptional activity assay in rice protoplasts. To evaluate the intrinsic transcriptional activity of each MoHTR, a $L U C$ reporter was expressed under the control of the basal expression promoter containing $5 \times$ GAL4 upstream activation sequence (UAS) and minimal $35 \mathrm{~S}$ promoter $\left(35 \mathrm{~S}_{\mathrm{mini}}\right)^{43}$. The effector vector was designed to produce MoHTR- $\Delta$ sp fused with GAL4 DNA-binding domain (GBD) for interaction with the $5 \times$ UAS (Supplementary Fig. 4). To evaluate the transcriptional activity of each MoHTR at the target gene promoters, a LUC reporter was expressed under the control of each target gene promoter. The effector vector was designed to produce MoHTR- $\Delta$ sp using the CaMV $35 \mathrm{~S}$ promoter and the TMV translation enhancer $(\Omega)$ sequence ${ }^{44}$ (Fig. 4a). The control vector that contains the GUS gene under the maize ubiquitin promoter was used for internal control.
The reporter, effector, and control vectors were introduced into rice protoplasts isolated from 2-week-old etiolated rice seedlings using a published protocol $^{42}$ with slight modification. Transfected protoplasts were incubated for 4 hours in W5 solution ( $\left.153 \mathrm{mM} \mathrm{NaCl}, 125 \mathrm{mM} \mathrm{CaCl}_{2}, 5 \mathrm{mM} \mathrm{KCl}, 2 \mathrm{mM} \mathrm{MES}\right)$, and their lysate was harvested using lysis buffer $[25 \mathrm{mM}$ Tris phosphate (pH 7.8), $2 \mathrm{mM}$ diaminocyclohexane tetraacetic acid, $10 \%$ glycerol, $1 \%$ Triton X-100, $2 \mathrm{mM}$ dithiothreitol]. A LUC reaction with the lysate was performed using the Luciferase Assay System from Promega. GUS assay using the cell lysate of transfected protoplasts was conducted in the reaction solution $[10 \mathrm{mM}$ Tris- $\mathrm{Hcl}(\mathrm{pH} 8.0), 2$ $\mathrm{mM} \mathrm{MgCl}, 1 \mathrm{mM}$ 4-Methylumbelliferyl- $B$-D-glucuronide ${ }^{45}$. The signal was quantified by the VICTOR3 multilabel plate reader (PerkinElmer). Normalized LUC activity was calculated by dividing the luminescence intensity by the GUS activity. The relative transcriptional activity of MoHTR was measured by comparing it with normalized LUC activity of the control reaction.

Genetic manipulation of $\boldsymbol{M}$. oryzae. M. oryzae field isolate KJ201 was obtained from the Center for Fungal Genetic Resources at Seoul National University. Fourday-old mycelia in complete medium broth were collected and treated with $3.3 \%$ lysing enzyme in $20 \%$ sucrose solution (w/v). After suspending generated protoplasts in STC [ $20 \%$ sucrose $(\mathrm{w} / \mathrm{v}), 50 \mathrm{mM}$ Tris- $\left.\mathrm{HCl}, 50 \mathrm{mM} \mathrm{CaCl}_{2}\right]$, they were used for PEG-mediated transformation. Knockout mutants were produced by replacing each target gene with a hygromycin phosphotransferase (HPH) cassette amplified from $\mathrm{pBCATPH}{ }^{46}$. Double-joint PCR was used to attach the $5^{\prime}$ and $3^{\prime}$ flanking regions of each target gene to the HPH cassette. Fungal transformants were selected using $200 \mathrm{ppm}$ hygromycin B. Gene deletion was confirmed by priority-based direct $\mathrm{PCR}^{47}$ and Southern analysis. Each gene with its own promoter was reintroduced to the corresponding knockout mutant using PEG-mediated transformation for complementation. A geneticin resistance gene cassette used for complementation was amplified from pII99 ${ }^{48}$. The complemented strains were selected on TB3 agar medium containing $800 \mathrm{ppm}$ geneticin. The primers used for fungal gene manipulation are listed in Supplementary Table 11.

Production of transgenic rice plants expressing each MoHTR gene without the signal peptide. MoHTR1- $\Delta$ sp and MoHTR2- $\Delta$ sp were amplified by PCR using the primers listed in Supplementary Table 11 and then integrated into the binary vector $\mathrm{pH} 2 \mathrm{GW}^{41}$ using the Gateway LR clonase (Invitrogen). The resulting constructs were introduced into the rice cultivar Nakdong via Agrobacterium tumefaciens-mediated transformation. LBA4404 harboring each construct was cultured in $\mathrm{AB}$ medium $\left(\mathrm{K}_{2} \mathrm{HPO}_{4} 3 \mathrm{~g} \mathrm{~L}^{-1}, \mathrm{NaH}_{2} \mathrm{PO}_{4} 1 \mathrm{~g} \mathrm{~L}^{-1}, \mathrm{NH}_{4} \mathrm{Cl} 1 \mathrm{~g} \mathrm{~L}^{-1}\right.$, $\mathrm{MgSO}_{4} 0.3 \mathrm{~g} \mathrm{~L}^{-1}, \mathrm{KCl} 0.15 \mathrm{~g} \mathrm{~L}^{-1}, \mathrm{CaCl}_{2} 7.5 \mathrm{mg} \mathrm{L}^{-1}, \mathrm{FeSO}_{4} 2.5 \mathrm{mg} \mathrm{L}^{-1}$ ) supplemented with $10 \mathrm{mg} \mathrm{L}^{-1}$ streptomycin and $50 \mathrm{mg} \mathrm{L}^{-1}$ hygromycin $\mathrm{B}$ for 3 days at $28^{\circ} \mathrm{C}$. Transgenic calli were selected on a medium containing $50 \mathrm{mg} \mathrm{L}^{-1}$ hygromycin $B$ and $250 \mathrm{mg} \mathrm{L}^{-1}$ cefotaxime. Rice plants were grown in a greenhouse under the $14 / 10$-h light/dark period, $24-28^{\circ} \mathrm{C}$, and $70-80 \%$ humidity.

Gene expression analysis via quantitative RT-PCR and RNA-Seq. Total rice RNA was isolated using the Easy-spin total RNA extraction kit (iNtRON Biotechnology) according to the manufacturer's instructions. First-strand cDNAs were synthesized using $2 \mu \mathrm{g}$ of total RNA and the ImProm-II Reverse Transcription System (Promega) with oligo (dT) primers. qRT-PCR reactions were performed using a Rotor-Gene Q real-time PCR cycler (Qiagen) and the gene-specific primers listed in Supplementary Table 11. Each PCR tube contained $5 \mu \mathrm{l}$ of $2 \times$ Rotor-Gene SYBR Green PCR master mix (Qiagen), $25 \mathrm{ng}$ of cDNA, and 15 pmol of each primer. The thermal cycling conditions were $10 \mathrm{~min}$ at $94^{\circ} \mathrm{C}$ followed by 40 cycles of $15 \mathrm{~s}$ at $94^{\circ} \mathrm{C}$ and $1 \mathrm{~min}$ at $60^{\circ} \mathrm{C}$

The quality of extracted RNA samples was assessed using Bioanalyzer 2100 (Agilent). RNA-Seq libraries were prepared using the TruSeq RNA Library Prep Kit (Illumina) and sequenced using HiSeq2500 (Illumina). Raw sequence reads were trimmed to remove adaptor sequences, and those with a quality lower than 20 were removed using the NGS QC Toolkit v2.3.349. All reads were assembled and mapped via the use of HISAT2 v2.1.0 $0^{50}$ and StringTie v1.3.5 $5^{51}$. DEGs in the MoHTR-OX lines were identified as the genes whose transcript abundance was $\geq 2$ fold higher or $\leq 0.5$-fold lower than their transcripts in Nakdong rice.

\section{Growth and developmental characteristics of MoHTR mutants. Vegetative} growth was checked by recording the diameter of each culture on complete agar medium (CM; $0.2 \%$ peptone, $1 \%$ glucose, $1 \%$ casamino acid, $0.1 \%$ yeast extract, $0.15 \% \mathrm{KH}_{2} \mathrm{PO}_{4}, 0.05 \% \mathrm{KCl}, 0.6 \% \mathrm{NaNO}_{3}, 0.05 \% \mathrm{MgSO}_{4}, 0.1 \%$ trace element, $0.1 \%$ vitamin supplement, and $1.5 \%$ agar, $\mathrm{pH} 6.5$ ) and minimal agar medium (MM; $0.15 \% \mathrm{KH}_{2} \mathrm{PO}_{4}, 0.05 \% \mathrm{KCl}, 0.6 \% \mathrm{NaNO}_{3}, 1 \%$ glucose, $0.05 \% \mathrm{MgSO}_{4}, 0.1 \%$ trace element, $0.1 \%$ vitamin supplement, and $1.5 \%$ agar, $\mathrm{pH} 6.5$ ). Conidiation was estimated by counting the number of conidia produced by 6 -day-old cultures on V8 agar in 6-well culture plate (SPL). All cultures were grown at $25^{\circ} \mathrm{C}$ under constant light. Conidial germination and appressoria formation were quantified at 12 hours after spotting a conidial suspension on hydrophobic coverslip.

Infection assays for fungal and bacterial pathogens. The rice cultivar Nakdong was grown in a growth chamber at $28^{\circ} \mathrm{C}$ and $80 \%$ humidity under a $16 \mathrm{~h}$-light $/ 8 \mathrm{~h}-$ dark photoperiod. After spray-inoculating 4-week-old plants with an M. oryzae 
conidial suspension $\left(5 \times 10^{4}\right.$ conidia/mL in $250 \mathrm{ppm}$ Tween20), the inoculated rice plants were incubated for 7 days. A numerical scoring system was employed to quantify disease: type 0 (no visible infection) to type 5 (large eyespot lesions, approximately $5 \mathrm{~mm}$ in length) ${ }^{52}$.

Six-week-old rice leaf sheaths were inoculated with an M. oryzae conidia suspension $\left(2 \times 10^{4}\right.$ conidia $/ \mathrm{mL}$ in sterile water). Inner epidermal layers of infected sheaths were excised and observed using a microscope. The disease severity was classified into four types based on the level of invasive fungal growth. Type 1 indicates that invasive hyphae remain in initially penetrated rice cells. Type 2 is defined as those that show the movement of invasive hyphae in initially infected cells to adjacent cells. In types 3 and 4 , invasive hyphae move further to the surrounding layers of cells from the previous type (Supplementary Fig. 7).

Barley seedlings were grown in a growth chamber at $28^{\circ} \mathrm{C}$ and $80 \%$ humidity under a $16 \mathrm{~h}$-light $/ 8 \mathrm{~h}$-dark photoperiod. M. oryzae conidial suspension $\left(5 \times 10^{4}\right.$ conidia/ml in $250 \mathrm{ppm}$ Tween20) was sprayed to 10 -day-old seedlings. The resulting lesions were observed and quantified after 5 days.

Five-week-old rice leaves were infected with Xoo K2, a strain compatible with cultivar Nakdong. The suspension of Xoo cells used for infection was prepared from colonies grown on nutrient agar at $28^{\circ} \mathrm{C}$ for three days. Rice leaves were inoculated with a bacterial suspension adjusted to $\mathrm{OD}_{600} 0.7$ using the scissor clip method $^{53}$. Lesion length was measured at $7 \mathrm{dpi}$.

Conidia of C. miyabeanus strain $\mathrm{Cm} 36$ were collected from 10-day-old culture on PDA. A conidial suspension $\left(1 \times 10^{3}\right.$ conidia/ml $)$ was prepared using $250 \mathrm{ppm}$ Tween 20 . Spray inoculated rice plants were incubated at $30^{\circ} \mathrm{C}$ and $85 \%$ humidity. Infected leaves were collected after 5 days. The lesion size was quantified using Image ${ }^{54}$.

Reporting summary. Further information on research design is available in the Nature Research Reporting Summary linked to this article.

\section{Data availability}

Data supporting the findings of this work are available within the paper and its Supplementary Information files. A reporting summary for this Article is available as a Supplementary Information file. The datasets and plant materials generated and analyzed during the current study are available from the corresponding author upon request. RNA-seq data has been deposited into the NCBI Sequence Read Archive under accession number PRJNA668035. Source data are provided with this paper.

Received: 7 May 2020; Accepted: 20 October 2020;

Published online: 17 November 2020

\section{References}

1. Jones, J. D. G. \& Dangl, J. L. The plant immune system. Nature 444, 323-329 (2006).

2. Bari, R. \& Jones, J. Role of plant hormones in plant defence responses. Plant Mol. Biol. 69, 473-488 (2009).

3. Pandey, S. P. \& Somssich, I. E. The role of WRKY transcription factors in plant immunity. Plant Physiol. 150, 1648-1655 (2009).

4. Khan, M., Seto, D., Subramaniam, R. \& Desveaux, D. Oh, the places they'll go! A survey of phytopathogen effectors and their host targets. Plant J. 93, 651-663 (2018).

5. Song, T. Q. et al. An oomycete CRN effector reprograms expression of plant HSP genes by targeting their promoters. PLoS Pathog. 11, el005348 (2015).

6. Yang, B., Sugio, A. \& White, F. F. Os8N3 is a host disease-susceptibility gene for bacterial blight of rice. Proc. Natl Acad. Sci. USA 103, 10503-10508 (2006).

7. Redkar, A. et al. A secreted effector protein of Ustilago maydis guides maize leaf cells to form tumors. Plant Cell 27, 1332-1351 (2015).

8. Qi, T. et al. Stripe rust effector PstGSRE1 disrupts nuclear localization of ROSpromoting transcription factor TaLOL2 to defeat ROS-induced defense in wheat. Mol. Plant 12, 1624-1638 (2019).

9. Vargas, W. A. et al. A fungal effector with host nuclear localization and DNAbinding properties is required for maize anthracnose development. Mol. Plant Microbe Interact. 29, 83-95 (2016).

10. Skamnioti, P. \& Gurr, S. J. Against the grain: safeguarding rice from rice blast disease. Trends Biotechnol. 27, 141-150 (2009).

11. Khang, C. H. et al. Translocation of Magnaporthe oryzae effectors into rice cells and their subsequent cell-to-cell movement. Plant Cell 22, 1388-1403 (2010).

12. Choi, J. et al. Fungal Secretome Database: integrated platform for annotation of fungal secretomes. BMC Genomics 11, 105 (2010).

13. Park, J. et al. FTFD: an informatics pipeline supporting phylogenomic analysis of fungal transcription factors. Bioinformatics 24, 1024-1025 (2008).

14. Horton, P. et al. WoLF PSORT: protein localization predictor. Nucleic Acids Res. 35, W585-W587 (2007)
15. Ba, A. N. N., Pogoutse, A., Provart, N. \& Moses, A. M. NLStradamus: a simple Hidden Markov Model for nuclear localization signal prediction. BMC Bioinformatics 10, 202 (2009).

16. Kosugi, S., Hasebe, M., Tomita, M. \& Yanagawa, H. Systematic identification of cell cycle-dependent yeast nucleocytoplasmic shuttling proteins by prediction of composite motifs. Proc. Natl Acad. Sci. USA 106, 10171-10176 (2009).

17. Jeon, J. et al. Transcriptome profiling of the rice blast fungus Magnaporthe oryzae and its host Oryza sativa during infection. Mol. Plant Microbe Interact. 33, 141-144 (2019).

18. Choi, J. et al. CFGP 2.0: a versatile web-based platform for supporting comparative and evolutionary genomics of fungi and Oomycetes. Nucleic Acids Res. 41, D714-D719 (2013).

19. Eom, K. S., Cheong, J. S. \& Lee, S. J. Structural analyses of zinc finger domains for specific interactions with DNA. J. Microbiol. Biotechnol. 26, 2019-2029 (2016).

20. Kelley, L. A., Mezulis, S., Yates, C. M., Wass, M. N. \& Sternberg, M. J. E. The Phyre2 web portal for protein modeling, prediction and analysis. Nat. Protoc. 10, 845-858 (2015)

21. Maiti, R., Van Domselaar, G. H., Zhang, H. \& Wishart, D. S. SuperPose: a simple server for sophisticated structural superposition. Nucleic Acids Res. 32, W590-W594 (2004)

22. Jones, K., Kim, D. W., Park, J. S. \& Khang, C. H. Live-cell fluorescence imaging to investigate the dynamics of plant cell death during infection by the rice blast fungus Magnaporthe oryzae. BMC Plant Biol. 16, 69 (2016).

23. Hossain, M. A. et al. The bZIP transcription factor OsABF1 is an ABA responsive element binding factor that enhances abiotic stress signaling in rice. Plant Mol. Biol. 72, 557-566 (2010).

24. Kim, M. J. et al. Quadruple 9-mer-based protein binding microarray with DsRed fusion protein. BMC Mol. Biol. 10, 91 (2009).

25. Liu, L. et al. An integrative bioinformatics framework for genome-scale multiple level network reconstruction of rice. J. Integr. Bioinform 10, 223 (2013).

26. Vannini, C. et al. The ectopic expression of the rice Osmyb4 gene in Arabidopsis increases tolerance to abiotic, environmental and biotic stresses. Physiol. Mol. Plant Pathol. 69, 26-42 (2006).

27. Shimono, M. et al. Rice WRKY 45 plays important roles in fungal and bacterial disease resistance. Mol. Plant Pathol. 13, 83-94 (2012).

28. Gomi, K. et al. Role of hydroperoxide lyase in white-backed planthopper (Sogatella furcifera Horváth)-induced resistance to bacterial blight in rice, Oryza sativa L. Plant J. 61, 46-57 (2010).

29. Deslandes, L. \& Rivas, S. The plant cell nucleus: a true arena for the fight between plants and pathogens. Plant Signal. Behav. 6, 42-48 (2011).

30. Buttner, D. \& Bonas, U. Getting across - bacterial type III effector proteins on their way to the plant cell. EMBO J. 21, 5313-5322 (2002).

31. Lo Presti, L. \& Kahmann, R. How filamentous plant pathogen effectors are translocated to host cells. Curr. Opin. Plant Biol. 38, 19-24 (2017).

32. Micali, C. O., Neumann, U., Grunewald, D., Panstruga, R. \& O'Connell, R. Biogenesis of a specialized plant-fungal interface during host cell internalization of Golovinomyces orontii haustoria. Cell. Microbiol 13, 210-226 (2011).

33. Kleemann, J. et al. Sequential delivery of host-induced virulence effectors by appressoria and intracellular hyphae of the phytopathogen Colletotrichum higginsianum. PLoS Pathog. 8, el002643 (2012).

34. Giraldo, M. C. et al. Two distinct secretion systems facilitate tissue invasion by the rice blast fungus Magnaporthe oryzae. Nat. Commun. 4, 1996 (2013).

35. Cermak, T. et al. Efficient design and assembly of custom TALEN and other TAL effector-based constructs for DNA targeting. Nucleic Acids Res. 39, e82 (2011).

36. Kloppholz, S., Kuhn, H. \& Requena, N. A secreted fungal effector of Glomus intraradices promotes symbiotic biotrophy. Curr. Biol. 21, 1204-1209 (2011).

37. Dean, R. A. et al. The genome sequence of the rice blast fungus Magnaporthe grisea. Nature 434, 980-986 (2005).

38. Sakai, H. et al. Rice Annotation Project Database (RAP-DB): an integrative and interactive database for rice genomics. Plant Cell Physiol. 54, e6 (2013).

39. Yao, W., Li, G. W., Yu, Y. M. \& Ouyang, Y. D. funRiceGenes dataset for comprehensive understanding and application of rice functional genes. Gigascience 7, gix119 (2017)

40. Gong, X. Y. et al. pFPL vectors for high-throughput protein localization in fungi: detecting cytoplasmic accumulation of putative effector proteins. Mol. Plant Microbe Interact. 28, 107-121 (2015).

41. Karimi, M., Inze, D. \& Depicker, A. GATEWAYTM vectors for Agrobacterium-mediated plant transformation. Trends Plant Sci. 7, 193-195 (2002).

42. Chen, S. B. et al. A highly efficient transient protoplast system for analyzing defence gene expression and protein-protein interactions in rice. Mol. Plant Pathol. 7, 417-427 (2006) 
43. Ohta, M., Ohme-Takagi, M. \& Shinshi, H. Three ethylene-responsive transcription factors in tobacco with distinct transactivation functions. Plant J. 22, 29-38 (2000).

44. Cho, J. I. et al. Role of the rice hexokinases OsHXK5 and OsHXK6 as glucose sensors. Plant Physiol. 149, 745-759 (2009).

45. Jefferson, R. A., Kavanagh, T. A. \& Bevan, M. W. GUS fusions: betaglucuronidase as a sensitive and versatile gene fusion marker in higher plants. EMBO J. 6, 3901-3907 (1987).

46. Yun, S. H., Turgeon, B. G. \& Yoder, O. C. REMI-induced mutants of Mycosphaerella zeae-maydis lacking the polyketide PM-toxin are deficient in pathogenesis to corn. Physiol. Mol. Plant Pathol. 52, 53-66 (1998).

47. Park, J., Kim, S., Kwon, S. \& Lee, Y. H. A quick and accurate screening method for fungal gene-deletion mutants by direct, priority-based, and inverse PCRs. J. Microbiol. Methods 105, 39-41 (2014).

48. Lee, J., Lee, T., Lee, Y. W., Yun, S. H. \& Turgeon, B. G. Shifting fungal reproductive mode by manipulation of mating type genes: obligatory heterothallism of Gibberella zeae. Mol. Microbiol 50, 145-152 (2003).

49. Patel, R. K. \& Jain, M. NGS QC Toolkit: a toolkit for quality control of next generation sequencing data. PLoS ONE 7, e30619 (2012).

50. Kim, D., Landmead, B. \& Salzberg, S. L. HISAT: a fast spliced aligner with low memory requirements. Nat. Methods 12, 357-360 (2015).

51. Pertea, M. et al. StringTie enables improved reconstruction of a transcriptome from RNA-seq reads. Nat. Biotechnol. 33, 290 (2015).

52. Valent, B., Farrall, L. \& Chumley, F. G. Magnaporthe grisea genes for pathogenicity and virulence identified through a series of backcrosses. Genetics 127, 87-101 (1991).

53. Park, C. J. et al. Overexpression of the endoplasmic reticulum chaperone $\mathrm{BiP} 3$ regulates XA21-mediated innate immunity in rice. PLoS ONE 5, e9262 (2010).

54. Schneider, C. A., Rasband, W. S. \& Eliceiri, K. W. NIH Image to ImageJ: 25 years of image analysis. Nat. Methods 9, 671-675 (2012).

\section{Acknowledgements}

This work was supported by grants from the National Research Foundation of Korea (NRF-2017R1A2A1A17069504, NRF-2015M3A9B8028679, NRF-2018R1A5A1023599, and NRF-2020R1A2B5B03096402) and the Korea Institute of Planning and Evaluation for Technology in Food, Agriculture, and Forestry through Agricultural Microbiome Program (918017-04). S. Kim and S. Kang are grateful for a graduate fellowship from the Brain Korea 21 Plus Program and a Brain Pool fellowship from the National Research Foundation of Korea (2019H1D3A2A01054562), respectively. We are grateful to Cecile Segonzac for valuable comments.

\section{Author contributions}

S.Kim and Y.-H.L. designed the research. S.Kim, C.-Y.K., S.-Y.P., H.C., and S.Kwon performed the experiments and analyzed the data. J.-S.J. generated transgenic rice plants. K.-T.K., Jongbum Jeon, G.C., and J.C. performed bioinformatics work. S.Kim, C.-Y.K., Junhyun Jeon, S.Kang, C.H.K., and Y.-H.L. wrote the article. All authors contributed to the editing of the manuscript.

\section{Competing interests}

The authors declare no competing interests.

\section{Additional information}

Supplementary information is available for this paper at https://doi.org/10.1038/s41467020-19624-w.

Correspondence and requests for materials should be addressed to Y.-H.L.

Peer review information Nature Communications thanks Ely Oliveira Garcia, and the other, anonymous, reviewer(s) for their contribution to the peer review of this work. Peer reviewer reports are available.

Reprints and permission information is available at http://www.nature.com/reprints

Publisher's note Springer Nature remains neutral with regard to jurisdictional claims in published maps and institutional affiliations.

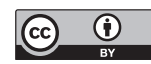

Open Access This article is licensed under a Creative Commons Attribution 4.0 International License, which permits use, sharing, adaptation, distribution and reproduction in any medium or format, as long as you give appropriate credit to the original author(s) and the source, provide a link to the Creative Commons license, and indicate if changes were made. The images or other third party material in this article are included in the article's Creative Commons license, unless indicated otherwise in a credit line to the material. If material is not included in the article's Creative Commons license and your intended use is not permitted by statutory regulation or exceeds the permitted use, you will need to obtain permission directly from the copyright holder. To view a copy of this license, visit http://creativecommons.org/ licenses/by/4.0/.

(C) The Author(s) 2020 